Received 00th January 20xx, Accepted 00th January 20xx DOI: $10.1039 / x 0 \times x 00000 x$

\section{The Law of Attraction: Relating Computed Energetics of Physisorption with Performance of Graphene-Based Sensors for Nitroaromatic Contaminants}

\begin{abstract}
Anna Piras ${ }^{\mathrm{a}}$ and Ganna Gryn'ova ${ }^{\mathrm{a} *}$
The ability to detect persistent nitroaromatic contaminants, e.g., DNT and TNT, with high sensitivity and selectivity is central to environmental science and medicinal diagnostics. Graphene-based materials rise to this challenge, offering supreme performance, biocompatibility, and low toxicity at a reasonable cost. In the first step of the electrochemical sensing process, these substrates establish non-covalent interactions with the analytes, which we show to be indicative of their respective detection limits. Employing a combination of semiempirical tight-binding quantum chemistry, meta-dynamics, density functional theory, and symmetry-adapted perturbation theory in conjunction with curated data from experimental literature, we investigate the physisorption of DNT and TNT on a series of functionalised graphene derivatives. In agreement with experimental observations, systems with greater planarity and positively charged substrates afford stronger noncovalent interactions than their highly oxidised distorted counterparts. Despite the highly polar nature of the investigated species, their non-covalent interactions are largely driven by dispersion forces. To harness these design principles, we considered a series of boron and nitrogen (co)doped two-dimensional materials. One of these systems featuring a chain of $\mathrm{B}-\mathrm{N}-\mathrm{C}$ units was found to adsorb nitroaromatic molecules stronger than the pristine graphene itself. These findings form the basis for the design principles of sensing materials and illustrate the utility of relatively low cost in silico procedures for testing the viability of designed graphene-based sensors for a plethora of analytes.
\end{abstract}

\section{INTRODUCTION}

From explosives testing grounds to oil deposits, from dye manufactures to ore mines, nitrogen-containing aromatic compounds (NACs), such as 2,4-dinitrotoluene (DNT) and 2,4,6trinitrotoluene (TNT) (Figure 1A), are omnipresent pollutants. ${ }^{1}$ For decades, these contaminants persist in soil and water, resisting biodegradation and posing a serious risk to human health due to their facile absorption through the skin and high bioaccumulation rates. $^{2}$ Consequently, detecting minute amounts of NACs in soil and water is of great importance to public health and safety, forensics, and anti-terrorism operations. ${ }^{3}$ Common modern detection methods include surface-enhanced Raman spectroscopy, ${ }^{4}$ high-performance liquid chromatography (HPLC), ${ }^{5}$ and numerous other analytical techniques. ${ }^{6-8}$ Among them, electrochemical detection based on the reduction of nitro-groups to amino-groups ${ }^{9}$ enables onsite real-time analysis, ${ }^{10}$ with the added benefits of low limits of detection (LOD), ${ }^{\ddagger}$ a large linear range, and a relatively low

\footnotetext{
a. Heidelberg Institute for Theoretical Studies (HITS g GmbH), SchlossWolfsbrunnenweg 35, 69118 Heidelberg, Germany, and Interdisciplinary Center for Scientific Computing (IWR), Heidelberg University, In Neuenheimer Feld 205, 69120 Heidelberg, Germany.E-mail: ganna.grynova@h-its.org

Electronic Supplementary Information (ESI) available: Additional discussion of the literature and analysis of the computed data, the full set of computed interaction energies, and absolute and relative electronic energies of all studied species (Part 1); Cartesian coordinates of the PBEO-D3-RIJCOSX/def2-TZVP optimised geometries for all studied species (Part 2). See DOI: 10.1039/x0xx00000x
}

cost of the apparatus. ${ }^{11}$ For these reasons, electrochemical sensors are among the most promising for the highperformance detection of NACs.

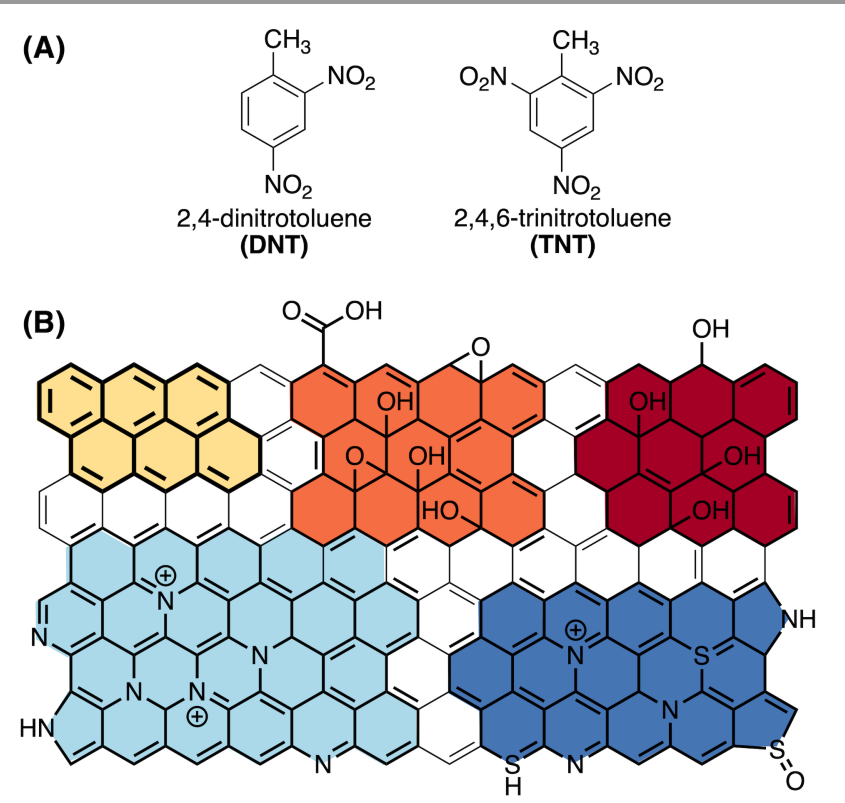

Figure 1. (A) Common NACs. (B) Structures of graphene-based materials: pristine graphene (bold yellow), reduced graphene oxide (orange), hydrogenated reduced graphene oxide (carmine), N-doped graphene (light blue) and $\mathrm{N}$ - and $\mathrm{S}$-(co)doped graphene (dark blue). 
The exceptional electrochemical ${ }^{12}$ and mechanical properties $^{13}$ of various graphene-based materials (GBMs, Figure 1B) make them particularly attractive to produce cheap, robust, and highly sensitive sensors. ${ }^{14}$ Two-dimensional GBM sensors enable the detection of a broad range of compounds, from amino acids to metal cations; ${ }^{15}$ such devices are also used for the subsequent removal of diverse aromatic contaminants from water. ${ }^{16-18}$ In 2014, reduced graphene oxide functionalised with 1,3,6,8-pyrenetetrasulfonic acid sodium salt and palladium nanoparticles was used to manufacture a sensor with a low limit of quantification of NAC explosives, ${ }^{19}$ however, this functionalised substrate is not economically viable for largescale production. Over the past decade, a variety of metal-free graphene-based materials - electrochemically exfoliated graphene, ${ }^{10}$ hydrogenated graphene, ${ }^{20}$ reduced graphene oxide, $^{21} \mathrm{~N}, \mathrm{~S}-(\mathrm{co})$ doped nanoribbons, ${ }^{22} \mathrm{~N}$-doped graphene ${ }^{23}$, and others ${ }^{24,25}$ - has been used to electrochemically detect DNT and TNT. Despite these formidable experimental efforts, a direct comparison between classes of materials is often obstructed by the disparate experimental conditions employed; thus, clear design principles grounded in a systematic understanding of NAC sensor chemistry are still lacking.

Across diverse sensor types, the initial interaction between an analyte and the sensor's surface is one of the key factors defining their detection efficiency. Physical adsorption via noncovalent interactions ( $\mathrm{NCls}$ ) affords faster sensor response and recovery compared to covalent binding between the 2D material and the analyte. ${ }^{15}$ In the case of $\mathrm{NO}_{2}$ detection, a quantitative relationship exists between the sensor's LOD and the computed adsorption energies and band gap of the graphene oxide sensing material. ${ }^{26}$ Similarly, the $\mathrm{NCls}$ are likely key to the detection of organic molecules with GBMs, since they influence the transport properties of a sensor, ${ }^{27,28}$ determine sensitivity and selectivity of the analytical procedure, ${ }^{15}$ and define the design principles for tailored carbon-based sensors. ${ }^{10,15}$ The nature of these interactions depends on the GBM and NAC structures: ${ }^{15}$ pristine graphene and its derivatives with a high degree of aromaticity interact with nitroaromatic compounds primarily via $\pi-\pi$ stacking, while functionalised graphene-derivatives (e.g., the reduced graphene oxide and the heteroatom-doped graphene) form additional hydrogen bonds and other electrostatic interactions. ${ }^{29}$ According to the results of one Fourier transform infrared spectroscopy study, the adsorption of nitroaromatic molecules is weaker on graphene oxide (due to its hydrophilicity and reduced aromaticity) and stronger on reduced graphene oxide (due to stacking with its $\pi$ system and electrostatic interactions with its numerous oxygenated functional groups). ${ }^{29}$ Several computational studies of the interactions between various organic molecules and graphene derivatives (see Table S1 in the ESI for a complete overview) led to similar observations. Once the nitroaromatic contaminant is adsorbed on the sensor surface, its electrochemical detection proceeds via a four-electron stepwise reduction of $\mathrm{NO}_{2}$ groups to amine functionalities. ${ }^{10}$ The ascertained electrochemical signal is thus largely determined by the electronic structure, i.e., the redox properties and conductivity of the sensor surface. Overall, both the initial adsorption and subsequent reduction of the analyte on the GBM sensor define the latter's efficiency, although the questions of whether either one of these factors dominates or if there is a strong correlation between them are yet to be fully clarified.

In this paper, we aim to determine whether there is a relationship between the strength and nature of the noncovalent interactions between nitroaromatic analytes and graphene-based sensors, quantified and analysed in silico, and the reported experimental effectiveness of these sensors. Should such a relationship exist, it would open doors to the targeted design of more sensitive and selective detector materials for a broader range of analytes; in case this relationship is weak or non-existent, the investigative focus should shift toward subsequent steps in the sensor's operation, i.e., the redox chemistry between GMBs and NACs responsible for the observed signal.

\section{COMPUTATIONAL METHODOLOGY}

The adsorption of DNT and TNT (Figure 1A) on various graphene substrates has been investigated in silico in the gas phase using a finite (nanoflake) model for graphene-based materials. Functionalised graphene derivatives of seven classes were constructed based on available experimental data, with an additional class representing materials yet to be explored, as follows (Table 1):

- Pristine graphene $(G)$.

- Reduced graphene oxide obtained with the Staudenmaier procedure (rGO-ST).

- Reduced graphene oxide obtained with the Hummers or Hofmann procedures ( $\mathrm{CGO}-\mathrm{HU}-\mathrm{HO}$ ).

- Graphene exfoliated in a $\mathrm{Na}_{2} \mathrm{SO}_{4}$ solution $\left(\mathrm{GO}-\mathrm{Na}_{2} \mathrm{SO}_{4}\right)$.

- Graphene exfoliated in a $\mathrm{LiClO}_{4}$ solution $\left(\mathrm{GO}-\mathrm{LiClO}_{4}\right)$.

- $\mathrm{N}$-doped graphene: this experimentally relevant system contains oxygenated groups introduced to the GBM structure during the doping process ${ }^{23}$ and is modelled by the $N$-doped$o x-G$ system. To elucidate the role of the nitrogen functional units alone in the interaction between $\mathrm{N}$-doped graphene and NACs, additional models containing only pyridinic nitrogen atoms ( $N$-doped-pyr-G) and graphitic and pyridinic nitrogen atoms ( $N$-doped-mix- $G$ ) were used.

- N,S-(co)doped graphene: similarly, several functionalised systems containing nitrogen and sulphur atoms in different oxidation states were considered ( $N, S$-codoped-thio-G, $N, S$ codoped-oxothio-G, and N,S-codoped-ox-G).

- B- and $\mathrm{N}$-(co)doped graphene: to additionally extend the established design guidelines beyond previously experimentally tested systems (classes 1-7 above), a series of graphene derivatives, increasingly (co)doped with boron and nitrogen atoms (BH-doped-G, NH-doped-G, B,N-codoped-Gn) up to hexagonal boron nitride $(h B N)^{30}$ were considered. To the best of our knowledge, these systems have not yet been applied to nitroaromatics sensing.

Pristine graphene was modelled as circumcoronene, while functionalised GBMs were modelled as substituted circumcoronenes with different distributions of functional 


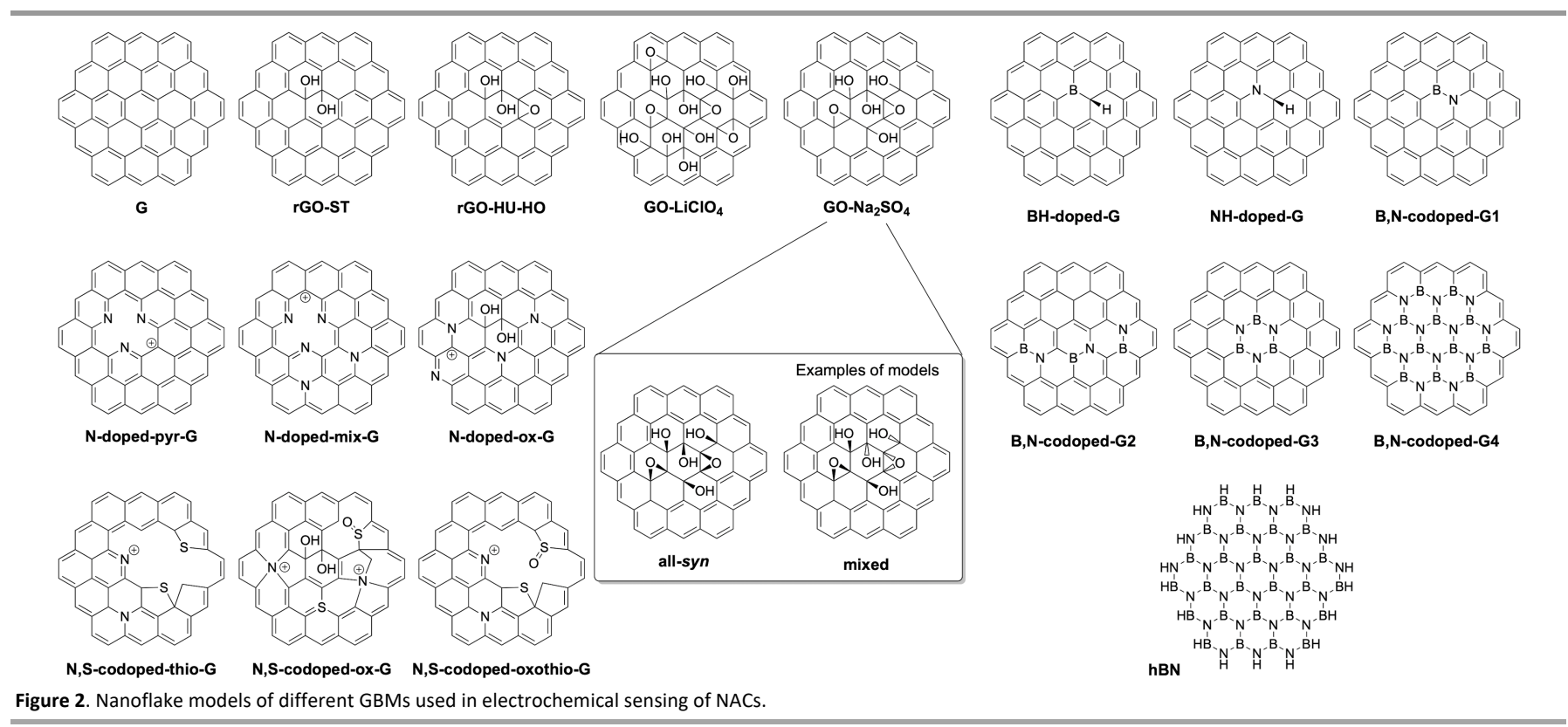

groups with respect to each other and the polyaromatic plane, i.e., all-syn and mixed (Figure 2). For doped graphene models, various types of heteroatomic functionalities were considered. Geometries of DNT, TNT, and GBM models were first preoptimised at a PBE-D3/def2-TZVP level of theory with the resolution of identity approximation (RIJCOSX) using ORCA. ${ }^{31}$

A semiempirical tight-binding quantum chemistry method (GFN2-xTB) was used in the framework of meta-dynamics $(\mathrm{MTD})^{32}$ with the root mean square deviation (RMSD) as a metric for collective variables to locate stable analyte-sensor complexes and compute their relative energies. Simulations were performed at $298 \mathrm{~K}$ for 20 picoseconds with a 1 femtosecond step using xtb software. ${ }^{32}$ For each complex, 400 frames were obtained. To maintain the planarity of the nanoflake models, they were constrained with a force constant of 10 Hartree; spherical logfermi potential was applied to avoid the dissociation of the non-covalent complexes. RMSDs were evaluated for 20 structures with a scaling value of 0.02 . These parameters were chosen as a good compromise between costly sampling of the potential energy surface and reasonable computational time. ${ }^{32}$

For each complex, structures within $20 \mathrm{~kJ} \mathrm{~mol}^{-1}$ of the lowest energy conformer at the MTD(RMSD)/GFN2-xTB level were chosen for a subsequent geometry and energy refinement at a PBEO-D3-RIJCOSX/def2-TZVP level of theory using ORCA. ${ }^{31}$ Accurate interaction energies of these complexes were obtained using symmetry-adapted perturbation theory (SAPT) $)^{33}$ at a SAPTO/jun-cc-pVDZ level with a density fitting approximation $^{34}$ as implemented in the Psi4 package. ${ }^{35}$ The same theory was employed to perform the energy decomposition analysis (EDA) of the computed interaction energies into physically meaningful components. ${ }^{36}$ The lowest energy (global minima) complexes are discussed further, while the full set of results is provided in the ESI. For selected complexes, the non-covalent interactions were additionally analysed using is a density-dependent scalar field, the densityoverlap regions indicator (DORI), which allows simultaneous characterisation of both covalent and non-covalent interactions. ${ }^{37}$ DORI was computed in conjunction with the PBEO-D3-RIJCOSX/def2-TZVP optimised geometries of the complexes at the PBEO-D3/DZP level of theory using ADF. ${ }^{38}$

\section{RESULTS AND DISCUSSION}

\subsection{Experimental data curation}

Table 1 provides an overview of the available literature data on the experimentally measured performance of diverse graphene-based sensors for nitroaromatic contaminants. The information collected refers not only to the limits of detection of DNT and TNT in various saline aqueous environments, but also, where available, to the analytical characterisation of GBM structure and composition, used in this work to construct the model systems.

We have analysed these experimental data to determine potential relationships between the adsorption of the analyte on the sensor's surface and the resulting detection efficacy, arriving at the following:

- Graphene obtained by electrochemical exfoliation: ${ }^{10}$ the limit of detection is generally lower for the GBMs obtained with the lithium perchlorate electrolyte relative to sodium sulphate. This trend is attributed to the higher content of oxygenated functional groups in $\mathrm{GO}-\mathrm{LiClO}_{4}(\mathrm{C} / \mathrm{O}$ ratio $=4)$ than in $\mathrm{GO}-\mathrm{Na}_{2} \mathrm{SO}_{4}$ $(\mathrm{C} / \mathrm{O}$ ratio $=8.8)$, which gives rise to stronger $\mathrm{NCls}$ between the sensor and the analyte and, ultimately, a lower limit of detection.

- $r G O$ and hydrogenated $r G O:{ }^{20}$ sensors employing nonhydrogenated $r G O$ enable improved detection relative to their hydrogenated analogues, highlighting the importance of sensor's planarity and $\pi$-conjugation, leading to stronger $\pi-\pi$ 
Table 1. Reported performance of graphene-based sensors of DNT and TNT.

\begin{tabular}{|c|c|c|c|c|c|}
\hline Ref. & NAC & Electrode $^{a}$ & Experimental conditions $^{b}$ & LOD, ppm & Composition (atomic content, functional groups, etc.) \\
\hline 10 & DNT & $\mathrm{GC}$ & BBS (pH 9.2) & 11.26 & / \\
\hline 10 & DNT & GC & 9:1 sea water $(\mathrm{pH} 8)$ and $\mathrm{BBS}(\mathrm{pH} 9.2)$ & 3.17 & / \\
\hline 20 & DNT & rGO & 9:1 sea water $(\mathrm{pH} 6.74)$ and $\mathrm{BBS}(\mathrm{pH} 9.2)$ & / & \\
\hline 20 & DNT & rGO-Hydr. & 9:1 sea water ( $\mathrm{pH} 6.74)$ and $\mathrm{BBS}(\mathrm{pH} 9.2)$ & / & Loss of aromaticity \\
\hline 21,39 & DNT & rGO-ST & BBS $(\mathrm{pH} 9.3)$ & / & $\mathrm{C} / \mathrm{O}=24.1$ \\
\hline 21,39 & DNT & rGO-HU & BBS (pH 9.3) & / & $\mathrm{C} / \mathrm{O}=19.27$ \\
\hline 21,39 & DNT & rGO-HO & BBS (pH 9.3) & / & $\mathrm{C} / \mathrm{O}=18.22$ \\
\hline 10 & DNT & $\mathrm{GO}_{-} \mathrm{LiClO}_{4}$ & 9:1 sea water $(\mathrm{pH} 8)$ and $\mathrm{BBS}(\mathrm{pH} 9.2)$ & 4.35 & $\mathrm{C} / \mathrm{O}=4 ;$ high in hydroxyls and carbonyls \\
\hline 10 & DNT & $\mathrm{GO}_{-} \mathrm{LiClO}_{4}$ & BBS $(\mathrm{pH} 9.2)$ & 2.73 & $\mathrm{C} / \mathrm{O}=4 ;$ high in hydroxyls and carbonyls \\
\hline 10 & DNT & $\mathrm{GO}-\mathrm{Na}_{2} \mathrm{SO}_{4}$ & 9:1 sea water $(\mathrm{pH} 8)$ and $\mathrm{BBS}(\mathrm{pH} 9.2)$ & 5.97 & $\mathrm{C} / \mathrm{O}=8.8$; epoxies, hydroxyls, carboxyls and carbonyls \\
\hline 10 & DNT & $\mathrm{GO}-\mathrm{Na}_{2} \mathrm{SO}_{4}$ & BBS $(\mathrm{pH} 9.2)$ & 5.43 & $\mathrm{C} / \mathrm{O}=8.8$; epoxies, hydroxyls, carboxyls and carbonyls \\
\hline 24 & TNT & G-sheet & 9:1 sea water $(\mathrm{pH} 8)$ and $\mathrm{BBS}(\mathrm{pH} 9.2)$ & 0.52 & $50 \times 50 \mathrm{~nm}$ exfoliation of G- nanofibers \\
\hline 24 & TNT & G-sheet & BBS & / & $50 \times 50 \mathrm{~nm}$ exfoliation of G- nanofibers \\
\hline 24 & TNT & G-ribbon & 9:1 sea water $(\mathrm{pH} 8)$ and $\mathrm{BBS}(\mathrm{pH} 9.2)$ & 0.14 & $5000 \times 300 \mathrm{~nm}$ (unzipping of CNTs) \\
\hline 24 & TNT & G-ribbon & BBS & / & $5000 \times 300 n m$ (unzipping of CNTs) \\
\hline 25 & TNT & G-SL & Artificial sea water & $\sim 1$ & Surface area $=\sim 10.000 \mathrm{~nm}^{2}$, length $=\sim 100 \mathrm{~nm}$ \\
\hline 25 & TNT & G-FL & Artificial sea water & $\sim 1$ & Surface area $=\sim 10.000 \mathrm{~nm}^{2}$, length $=\sim 100 \mathrm{~nm}$ \\
\hline 25 & TNT & G-ML & Artificial sea water & $\sim 1$ & Thickness $=\sim 5 \mathrm{~nm}$, length $=\sim 100 \mathrm{~nm}$ \\
\hline 25 & TNT & $\begin{array}{l}\text { Graphite micro- } \\
\text { particles }\end{array}$ & Artificial sea water & $\sim 1$ & Diameter $=\sim 10-20 \mu \mathrm{m}$ \\
\hline 20 & TNT & rGO & 9:1 sea water ( $\mathrm{pH} 6.74)$ and $\mathrm{BBS}(\mathrm{pH} 9.2)$ & 0.40 & \\
\hline 20 & TNT & rGO-Hydr. & 9:1 sea water ( $\mathrm{pH} 6.74)$ and $\mathrm{BBS}(\mathrm{pH} 9.2)$ & 0.50 & Loss of aromaticity \\
\hline 21,39 & TNT & rGO-ST & BBS $(\mathrm{pH} 9.3)$ & / & $\mathrm{C} / \mathrm{O}=24.1$ \\
\hline 21,39 & TNT & rGO-HO & 9:1 sea water $(\mathrm{pH} 8)$ and $\mathrm{BBS}(\mathrm{pH} 9.3)$ & / & $\mathrm{C} / \mathrm{O}=18.22$ \\
\hline 21,39 & TNT & rGO-HO & BBS $(\mathrm{pH} 9.3)$ & / & $\mathrm{C} / \mathrm{O}=18.22$ \\
\hline 21,39 & TNT & rGO-HU & BBS $(\mathrm{pH} 9.3)$ & / & $C / O=19.27$ \\
\hline 10 & TNT & $\mathrm{GO}-\mathrm{LiClO}_{4}$ & 9:1 sea water $(\mathrm{pH} 8)$ and $\mathrm{BBS}(\mathrm{pH} 9.3)$ & 2.03 & $\mathrm{C} / \mathrm{O}=4 ;$ high in hydroxyls and carbonyls \\
\hline 10 & TNT & $\mathrm{GO}-\mathrm{LiClO}_{4}$ & BBS $(\mathrm{pH} 9.2)$ & 6.74 & $\mathrm{C} / \mathrm{O}=4 ;$ high in hydroxyls and carbonyls \\
\hline 10 & TNT & $\mathrm{GO}-\mathrm{Na}_{2} \mathrm{SO}_{4}$ & 9:1 sea water $(\mathrm{pH} 8)$ and $\mathrm{BBS}(\mathrm{pH} 9.2)$ & 3.85 & $\mathrm{C} / \mathrm{O}=8.8$; epoxies, hydroxyls, carboxyls and carbonyls \\
\hline 10 & TNT & $\mathrm{GO}-\mathrm{Na}_{2} \mathrm{SO}_{4}$ & BBS $(\mathrm{pH} 9.2)$ & 6.54 & $\mathrm{C} / \mathrm{O}=8.8$; epoxies, hydroxyls, carboxyls and carbonyls \\
\hline 23 & TNT & $\begin{array}{l}\text { N-doped } \\
\text { graphene }\end{array}$ & Artificial sea water degassed & 0.03 & Mainly pyridine-like $\mathrm{N}$ \\
\hline 22 & TNT & $\begin{array}{l}\text { N,S-(co)doped } \\
\text { rGO nanoribbon }\end{array}$ & $0.1 \mathrm{M}$ PBS (pH 5.5) with $0.4 \mathrm{M} \mathrm{KCl}$ & 0.0001 & $\begin{array}{l}\text { Oxygen containing groups, pyridinic and pyrrolic } \mathrm{N}, \\
\text { thiophene and oxidised thiophene }\end{array}$ \\
\hline
\end{tabular}

${ }^{a}$ Electrode notations: $\mathrm{GC}$ - glassy carbon electrode; $\mathrm{GO}-\mathrm{LiClO}_{4}$ - graphene obtained by electrochemical exfoliation with lithium perchlorate; $\mathrm{GO}_{-} \mathrm{Na}_{2} \mathrm{SO}_{4}-$ graphene obtained by electrochemical exfoliation with sodium sulphate; $r G O$ - obtained by thermal reduction of graphite oxide in an argon atmosphere; $r G O-H y d r$. - obtained by thermal reduction of graphite oxide in a saturated $\mathrm{H}_{2}$ atmosphere; $\mathrm{rGO}-\mathrm{ST}$ - thermally reduced graphene oxide obtained with $\mathrm{Staudenmaier}$ procedure; ${ }^{24} \mathrm{rGO}$ - $\mathrm{HO}-$ thermally reduced graphene oxide obtained with the Hofmann procedure; ${ }^{24} \mathrm{rGO}-\mathrm{HU}$ - thermally reduced graphene oxide obtained with Hummers procedure; G-SL single-layered graphene nanoribbons; $G-F L$ - few-layered graphene nanoribbons; $G-M L$ - multi-layered graphene nanoribbons.

${ }^{b}$ Experimental conditions notations: $B B S$ - borate-buffered saline; artificial sea water $-0.5 \mathrm{M} \mathrm{NaCl}$ solution; $P B S-$ phosphate-buffered saline.

stacking with the nitroaromatic analytes.

- Graphene nanosheets and nanoribbons: ${ }^{24}$ detectors utilising nanoribbons, rich in $\mathrm{sp}^{2}$ carbon, are more sensitive than those based on nanosheets, further emphasising the importance of the $\pi-\pi$ stacking with the analyte.

- rGO obtained with different procedures: according to Ref. 21 , the sensitivity of the sensor depends on the oxygen content in the graphene-based material. Specifically, in the series $r G O$ $H O, r G O-H U, r G O-S T$, the sensitivity of the detector improves with decreasing load of oxygen-containing functional groups.

- Single-, few-, multi-layered nanoribbons and graphite nanoparticles: ${ }^{25}$ sensitivity of these sensors is only weakly dependent on the number of graphene layers, while the linear range of response is larger for graphite nanoparticles.
- $N$-doped graphene:23 these sensors afford low limits of detection, potentially due to additional non-covalent interactions between the electron-poor nitroaromatic analyte and the electron-rich graphene derivative containing pyridinic nitrogen atoms.

- $N, S-(c o)$ doped nanoribbons: ${ }^{22}$ these sensors demonstrate the best performance, likely due to additional hydrogen bonding between the sensor's functionalities and the analyte. However, synthesising such materials is complex, lengthy, and costly.

\subsection{Structures of NAC-GBM complexes}

Several finite models of graphene-based sensing materials from classes 1 to 7 were chosen for in silico exploration (Figure 2). 
Their structures sans analyte were optimised at the PBEO-D3RIJCOSX/def2-TZVP level of theory. To quantitatively assess the extent of the GBM's planarity in the relaxed structures, we employed a geometrical measure of puckering in the parent circumcoronene ring (see Figure S1 and Table S2 in the ESI). ${ }^{40}$ Optimised geometries of the studied GBM models display the following features:

- Pristine graphene (class 1 , circumcoronene model $G$ ) is a planar $\pi$-conjugated system.

- Reduced graphene oxide (classes 2 and 3, rGO-ST, rGO-HUHO): a low degree of oxidation is introduced and the planarity and $\pi$-conjugation within the system is partially lost.

- Exfoliated graphene oxide (classes 4 and 5, GO-Na $\mathrm{SO}_{4}, \mathrm{GO}$ $\mathrm{LiClO}_{4}$ ): due to the high extent of oxidation and presence of many $\mathrm{sp}^{3}$ carbons, the planarity is significantly depleted, particularly in the case of all-syn models.

- $N$-doped graphene (class 6, N-doped-ox-G, N-doped-pyr-G, and $N$-doped-mix-G): model GBMs containing only nitrogen atoms are planar $\pi$-conjugated systems, while the low degree of oxidation introduced in the $\mathrm{N}$-doped-ox- $\mathrm{G}$ causes partial loss of planarity.

- $\quad$ N,S-(co)doped graphene (class 7, N,S-codoped-thio-G, N,Scodoped-oxothio-G, and $N, S$-codoped-ox-G): the presence of defects and $\mathrm{sp}^{3}$ carbon atoms distorts the planarity and bonding pattern in the circumcoronene skeleton, particularly in the case of the oxidised species.
Next, 30 complexes of model graphene derivatives with DNT and TNT analytes were investigated at the MTD(RMSD)/GFN2xTB level. Multiple stable conformations for each complex were located and subsequently refined at the PBEO-D3RIJCOSX/def2-TZVP level. Finally, they were ranked according to electronic energies at the same level of theory. The threedimensional structures of these lowest-energy NAC-GBM complexes are shown in Figure 3 below.

In all investigated systems, the nature of the interactions between NACs and GBMs is physisorption, i.e., no directional covalent bonding is established. In the case of highly planar nanoflakes, the NAC molecule is adsorbed parallel to the circumcoronene plane, while distorted GBMs give rise to tilted adsorption geometries. The bonding pattern of the nanoflake is generally maintained in the adsorption complexes, except for the $N, S$-codoped-thio-G system, in which the connectivity changes in the defect-rich zone upon interaction with the analyte.

\subsection{Non-covalent interactions in NAC-GBM complexes}

Analyte-surface interaction energies in the located stable complexes were further refined using symmetry-adapted perturbation theory, which is considered to provide reliable energetics for non-covalent interactions (see Figure S2 in the ESI, which shows good qualitative agreement between PBEO and SAPTO results). ${ }^{33,34}$ According to our results (Table 2 ), the
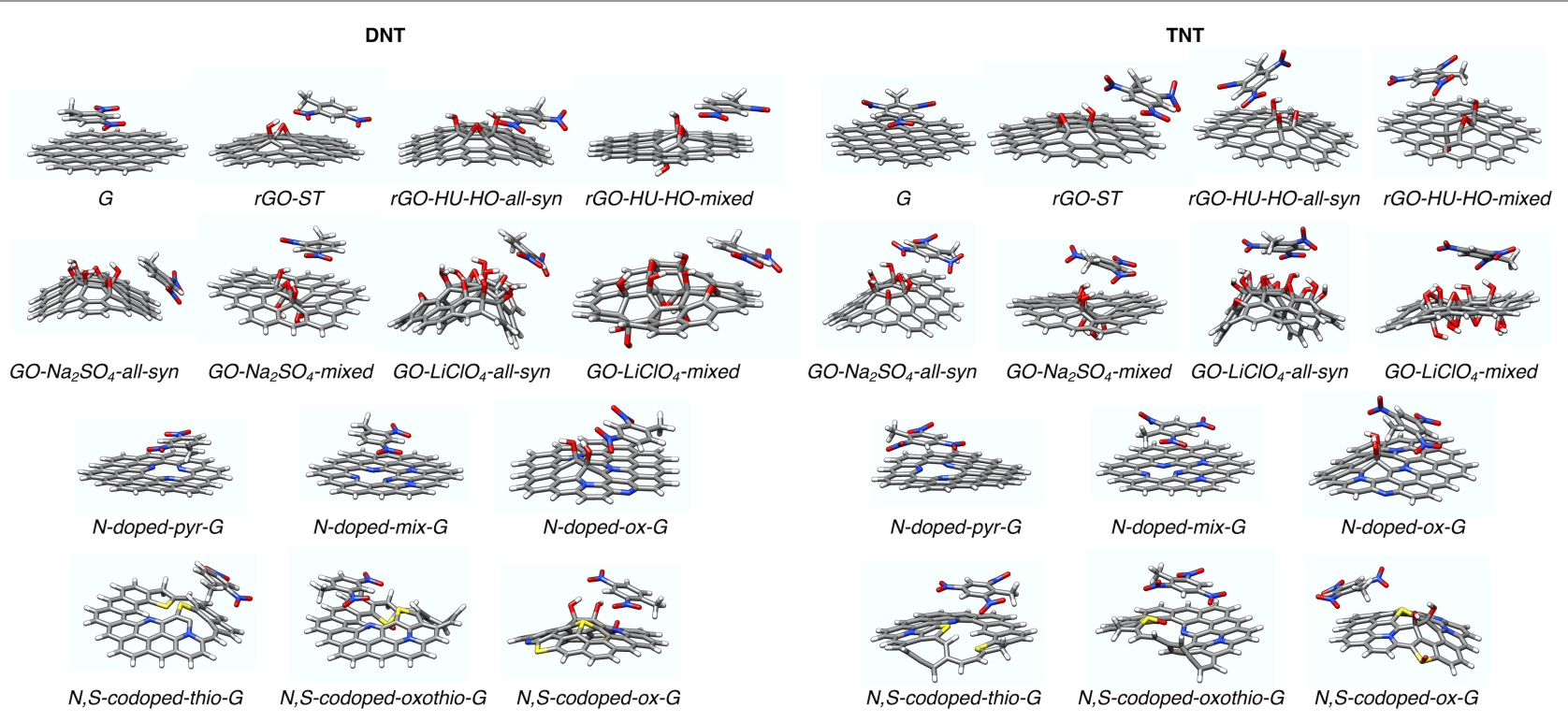

GO-Na $\mathrm{SO}_{4}$-all-syn

GO- $\mathrm{Na}_{2} \mathrm{SO}_{4}$-mixed $\mathrm{GO}-\mathrm{LiClO}_{4}$-all-syn

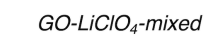

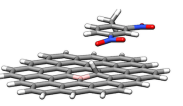

BH-doped-G

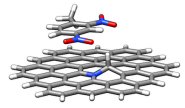

NH-doped-G

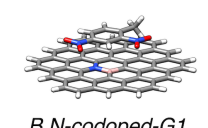

B,N-codoped-G1

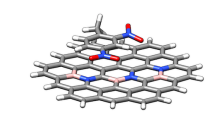

B,N-codoped-G2

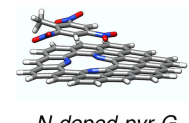

$N$-doped-pyr-G

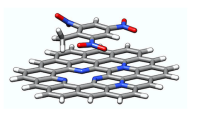

$N$-doped-mix-G
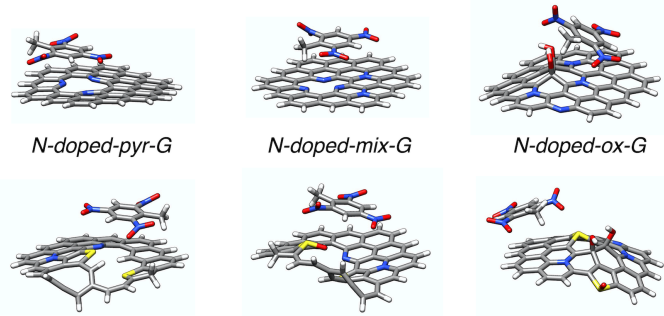

N,S-codoped-thio-G

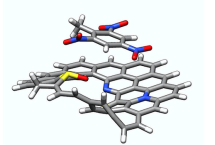

N,S-codoped-oxothio-G

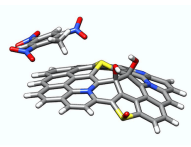

N,S-codoped-ox-G

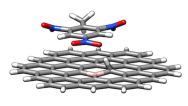

BH-doped-G

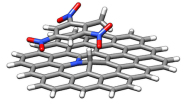

NH-doped-G

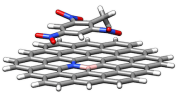

B,N-codoped-G1

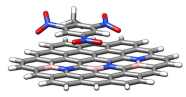

$B, N$-codoped-G2

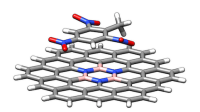

B,N-codoped-G3

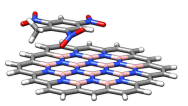

B,N-codoped-G4

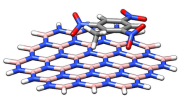

$h B N$

Figure 3. Three-dimensional structures of the most stable adsorption geometries of the DNT-GBM and TNT-GBM complexes (PBEO-D3/def2-TZVP). 
interaction energies between the nitroaromatic pollutants and various investigated graphene derivatives from classes 1 to 7 fall between -140 and $-60 \mathrm{~kJ} \mathrm{~mol}^{-1}$, and generally follow similar trends for DNT and TNT (see Figure S3 in the ESI). The interaction distances between NACs and GBMs in their complexes average around 3.3-3.4 $\AA$; interestingly, no appreciable correlation is observed between the strength and the spatial range of the studied interactions (see Figure S4 in the ESI). Relative to adsorption on pristine graphene, stronger interactions are established with positively charged GBMs ( $N$ doped and $\mathrm{N}, \mathrm{S}$-codoped substrates), while multiple oxygenated functionalities ( $\mathrm{GO}-\mathrm{Na}_{2} \mathrm{SO}_{4}$ and $\left.\mathrm{GO}-\mathrm{LiClO}_{4}\right)$ result in weakened adsorption. Furthermore, GBMs with a higher extent of planarity in the $\pi$-conjugated core ensure better adsorption of NACs (Figure 4A). These theoretical observations are in good agreement with the factors that determine the experimental performance of the GBM sensors discussed above.

Table 2. SAPTO/jun-cc-pVDZ interaction energies $\left(\triangle E_{\text {SAPTO }}, \mathrm{kJ} \mathrm{mol}^{-1}\right)$ and intermolecular interaction distances $(d, \AA)^{a}$ in the PBEO-D3/def2-TZVP global minima for the studied NAC-GBM complexes.

\begin{tabular}{|c|c|c|c|c|c|}
\hline & \multirow[t]{2}{*}{ Model GBM } & \multicolumn{2}{|c|}{ DNT } & \multicolumn{2}{|c|}{ TNT } \\
\hline & & $\Delta E_{\text {SAPTO }}$ & $d, \AA$ & $\Delta E_{\text {SAPTO }}$ & $d, \AA$ \\
\hline 1 & $G$ & -104.8 & 3.43 & -119.9 & 3.41 \\
\hline 2 & $r G O-S T$ & -92.5 & 3.62 & -86.7 & 3.37 \\
\hline 3 & rGO-HU-HO-all-syn & -85.5 & 3.40 & -95.6 & 2.95 \\
\hline 3 & rGO-HU-HO-mixed & -91.7 & 3.37 & -111.2 & 3.24 \\
\hline 4 & $\mathrm{GO}-\mathrm{Na}_{2} \mathrm{SO}_{4}$-all-syn & -96.4 & 3.24 & -102.6 & 2.94 \\
\hline 4 & $\mathrm{GO}-\mathrm{Na}_{2} \mathrm{SO}_{4}$-mixed & -86.7 & 3.40 & -94.4 & 2.88 \\
\hline 5 & GO-LiClO 4 -all-syn & -81.3 & 3.19 & -107.5 & 3.04 \\
\hline 5 & GO-LiClO 4 -mixed & -80.3 & 3.07 & -64.6 & 3.08 \\
\hline 6 & $N$-doped-ox-G & -92.1 & 3.49 & -97.0 & 3.20 \\
\hline 6 & $N$-doped-pyr-G & -106.6 & 3.64 & -114.5 & 3.38 \\
\hline 6 & $N$-doped-mix-G & -141.3 & 3.36 & -139.0 & 3.28 \\
\hline 7 & $N, S$-codoped-ox-G & -75.0 & 3.62 & -82.3 & 3.38 \\
\hline 7 & N,S-codoped-thio-G & -87.6 & 3.46 & -101.5 & 3.32 \\
\hline 7 & N,S-codoped-oxothio-G & -102.5 & 3.59 & -108.0 & 3.35 \\
\hline 8 & BH-doped-G & -98.7 & 3.55 & -107.7 & 3.49 \\
\hline 8 & NH-doped-G & -98.7 & 3.42 & -80.1 & 3.54 \\
\hline 8 & $B, N$-codoped-G1 & -105.7 & 3.36 & -114.6 & 3.39 \\
\hline 8 & $B, N$-codoped-G2 & -114.3 & 3.37 & -121.2 & 3.35 \\
\hline 8 & $B, N$-codoped-G3 & -98.4 & 3.32 & -106.5 & 3.40 \\
\hline 8 & $B, N$-codoped-G4 & -83.5 & 3.38 & -99.2 & 3.27 \\
\hline 8 & $h B N$ & -65.4 & 3.41 & -73.7 & 3.48 \\
\hline
\end{tabular}

${ }^{a}$ Interaction distance is evaluated as the distance between the carbon atom of the NAC phenyl ring and the closest non-hydrogen atom of the GBM.

A complete picture of non-covalent interactions between nitroaromatic contaminants and graphene-based sensors encompasses not only structural and total interaction energy features, but also an energy decomposition analysis of the latter (see Figure S5 in the ESI). Dispersion constitutes the largest attractive - and, ultimately, the definitive - contribution to the SAPTO total interaction energies of the studied NCls and is only partially countered by exchange repulsion. Moreover, dispersion is greatest in complexes formed by more planar substrates - pristine and $\mathrm{N}$-doped graphenes - due to closer contact between the analyte and the sensor surface. Interestingly, the electrostatic component is stabilising in all systems - both neutral and positively charged - and is a combination of several effects: (i) $\pi-\pi$ interactions between electron-deficient NACs and graphene's $\pi$-system, and (ii) electrostatic interactions between nitro-groups and functional units (substituents and dopant atoms) in the GBM. The nature of these interactions - attractive or repulsive - is specific to a given complex; however, as noted before, electrostatic effects are secondary to dispersion in all studied systems.
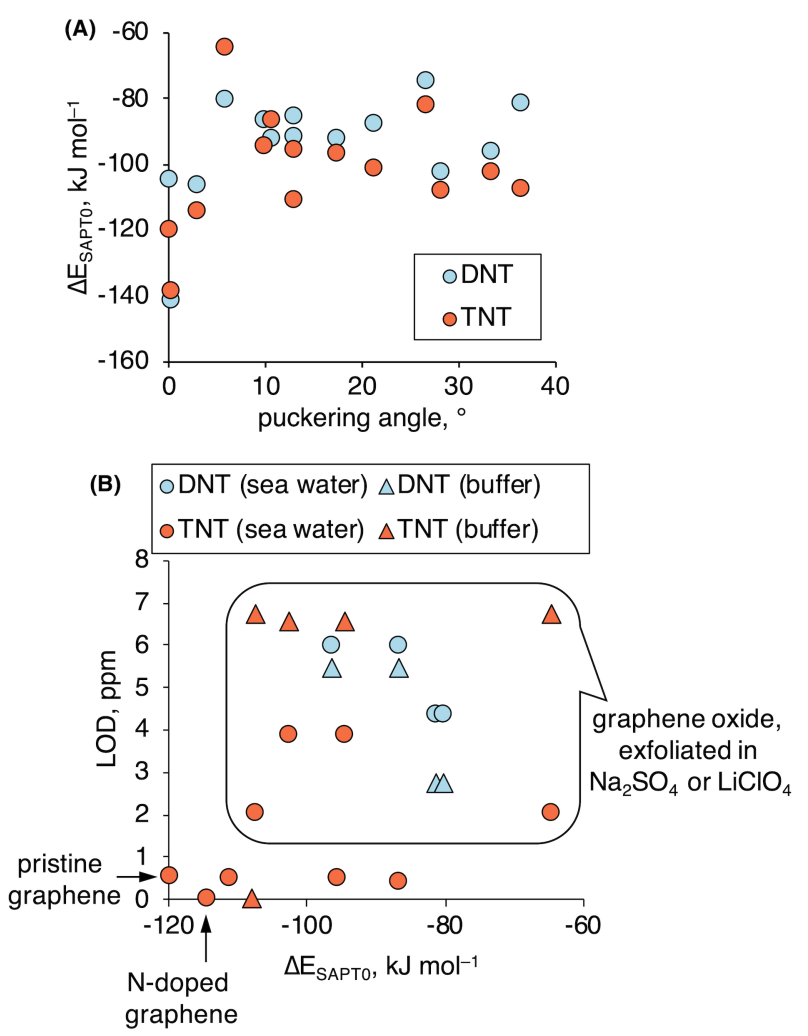

Figure 4. (A) The relationship between SAPTO/jun-cc-pVDZ interaction energies of NACGBM complexes and planarity of the corresponding graphene substrates. (B) Experimentally measured limits of detection of DNT and TNT in saline aqueous environments (Table 1) plotted against computed SAPTO/jun-cc-pVDZ interaction energies in NAC-GBM complexes (Table 2).

\subsection{The role of adsorption in the overall sensing performance}

Does the strength of the initial adsorption of the analyte on the sensor surface directly define the resulting sensor performance? To address this question, we explored the relationship between the computed interaction energies of the nitroaromatic pollutants with model graphene-based materials on one side and the experimentally determined limits of detection of the corresponding electrochemical sensors on the other (Figure 4B). Such an analysis is limited by the availability of experimental data and its homogeneity, i.e., the differences in the experimental conditions. Furthermore, computed results correspond to finite models of graphene sensors in vacuo and therefore do not capture the full complexity of the real-life systems. Nonetheless, there is overall qualitative agreement between the $\mathrm{NCl}$ strengths and the sensor's limits of detection: highly planar and positively charged GBMs afford stronger adsorption and result in lower LODs, while exceedingly 
distorted graphene oxide materials carrying multiple oxygenated functional groups lead to weaker adsorption and poorer sensor performance. Interestingly, the N,S-(co)doped substrate, which demonstrated outstanding sensing of TNT in a phosphate-buffered saline (Table 1), does not establish the strongest interactions with NACs compared to other tested GBMs. This potentially indicates the limitations of comparing the results of experimental studies conducted under conditions that are too dissimilar; based on our computational data alone, expensive and challenging to synthesise (co)doped graphenes are unlikely to substantially outperform cheaper and simpler analogues.

\subsection{Performance of $\mathrm{B}, \mathrm{N}-(\mathrm{co})$ doped GBMs}

Armed with the established relationships between the detection limit of a sensor, planarity of the sensing material, and the strength of its non-covalent interactions with the analyte, we proceeded to investigate another class (class 8 in section 2) of graphene-like materials doped with boron and nitrogen atoms. Systems with distinct quantities and arrangements of B and $\mathrm{N}$ atoms within the graphene lattice, as well as the fully doped graphene analogue, flat hexagonal boron nitride ( $h B N$, Figure 2), were investigated using the computational approach described above. These species generally retain their planarity upon physisorption of the nitroaromatic molecule (Figure 3 ), while the interaction energies in the formed complexes, computed at the SAPT0/jun-cc-pVDZ level of theory, fall within the range of -120 to $-60 \mathrm{~kJ} \mathrm{~mol}^{-1}$ (Table 2). Interestingly, the strongest interactions are achieved with the species $B, N$ codoped-G2, which contains a chain sequence of $\mathrm{B}-\mathrm{N}-\mathrm{C}$ units; arranging $\mathrm{B}$ and $\mathrm{N}$ atoms in a ring (e.g. $B, N$-codoped-G3) and/or increasing the dopant content (e.g. B,N-codoped-G4) weakens NAC's physisorption, and the $\triangle E_{S A P T O}$ for the flat hexagonal boron nitride is only $c a .-70 \mathrm{~kJ} \mathrm{~mol}^{-1}$. Similar to the systems from classes 1-7, dispersion is the main driving force behind the analyte-sensor interactions (see Figure S5 in the ESI). To further analyse the peculiar interactions afforded by $B, N$-codoped-G2, the domains of electron density overlaps were visualised and analysed using DORI for the DNT and TNT adsorbed on this system, as well as pristine graphene $G$ and hexagonal boron nitride $h B N$ (Figure 5, see also frontier molecular orbital plots in Figure $\mathrm{S} 6$ of the ESI). All of these complexes involve a single pronounced DORI domain between the analyte and basal plane of the material. The most pronounced feature of the $B, N$ codoped-G2 complexes is the electron density overlap between the methyl group of the NAC and the GBM surface, notably greater compared to the reference $G$ and $h B N$ complexes. This suggests that the propensity to establish dispersion-driven $\mathrm{CH}-$ $\pi$ interactions is enhanced in systems involving particular $\mathrm{B}-\mathrm{N}-$ $C$ patterns, found in the $B, N$-codoped-G2 material.

\section{CONCLUSIONS}

The non-covalent interactions responsible for the physisorption of nitroaromatic pollutants on the surface of graphene-based electrochemical sensors are a crucial factor defining detector performance. Employing a combination of semiempirical tight- binding in the framework of meta-dynamics to explore the structural space of physisorption with density functional theory and symmetry-adapted perturbation theory to assess its energetics, we have analysed these interactions for a series of finite models of various graphene-based materials with the prototypical nitroaromatic contaminants, dinitro- and trinitrotoluene. In general, GBMs capable of stronger analyte binding are characterised by lower detection limits. The interaction energy is found to depend on the extent of the substrate's planarity and to be largely driven by dispersion rather than electrostatic interactions. Stronger adsorption and lower LODs are found in sensors featuring pristine and N-doped graphene, while highly oxidised non-planar derivatives demonstrate relatively weaker binding and poorer performance. Moreover, analysis of the geometric and electronic structure of the sensor material alone provides useful insights into its detection potential at a comparatively low computational cost. These findings forge a path toward deeper understanding of the fundamental physical forces underlying electrochemical sensing with GBMs, which lays the groundwork for conceptual design guidelines of better sensors for a wider scope of analytes. As a first step on this path, we investigated the non-covalent interactions of nitroaromatic molecules with boron- and nitrogen-(co)doped GBMs, which to date have not been tested for the detection of NACs. A substrate containing a chain pattern of $\mathrm{B}-\mathrm{N}-\mathrm{C}$ units within the graphene lattice yielded interaction energy values approximately $20 \mathrm{~kJ} \mathrm{~mol}^{-1}$ stronger than for NACs on pristine graphene. At the same time, flat hexagonal boron nitride was found to provide only intermediate stabilisation of the analyte on its surface.

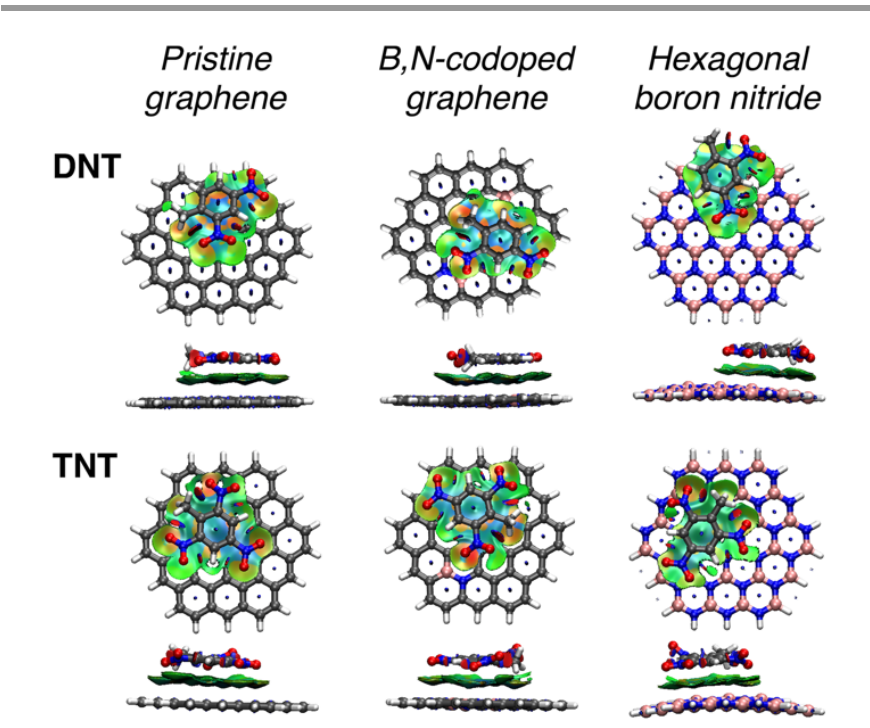

Figure 5. DORI $=0.98$ isosurfaces for selected NAC-GBM complexes, views from above and along the basal plane of the GBM model. Isosurfaces are colour-coded with $\operatorname{sgn}\left(\lambda_{2}\right) \rho(\mathbf{r})$ in the range from -0.01 au (red) to 0.01 au (blue).

While this study sheds light on the role of non-covalent interactions in the sensing process, our conclusions are subject to several caveats. On one hand, more homogeneous experimental data is necessary to deduce clearer relationships between a given sensor's physico-chemical properties and its 
sensitivity. On the other hand, further theoretical assessments are needed to estimate the validity of the finite models of sensing materials, 14 evaluate the contribution of environmental effects (e.g., solvation and applied external electric fields), and elucidate the importance of the subsequent steps in electrochemical sensing (i.e., the redox properties of the GBM). To enable these future steps, this work provides a solid starting point toward a deeper understanding of how the chemistry and physics of graphene-based materials in environmental sensing applications can be rationalised and modulated in silico.

\section{Author Contributions}

Anna Piras: investigation, methodology, data curation, formal analysis, visualisation, writing - original draft. Ganna Gryn'ova: conceptualisation, supervision, formal analysis, visualisation, writing - review \& editing.

\section{Conflicts of interest}

11 K.-S. Ju and R. E. Parales, Nitroaromatic Compounds, from Synthesis to Biodegradation, Microbiol. Mol. Biol. Rev., 2010, 74, 250-272, DOI: 10.1128/mmbr.00006-10.

22 S. Letzel, T. Göen, M. Bader, J. Angerer and T. Kraus, Exposure to Nitroaromatic Explosives and Health Effects during Disposal of Military Waste, Occup. Environ. Med., 2003, 60, 483-488, DOI: 10.1136/oem.60.7.483.

$3{ }^{3} \mathrm{~S}$. Singh, Sensors-An Effective Approach for the Detection of Explosives, J. Hazard. Mater., 2007, 144, 15-28, DOI: 10.1016/j.jhazmat.2007.02.018.

44 L. C. Pacheco-Londoño, W. Ortiz-Rivera, O. M. PrimeraPedrozo and S. P. Hernández-Rivera, Vibrational Spectroscopy Standoff Detection of Explosives, Anal. Bioanal. Chem., 2009, 395, 323-335, DOI: 10.1007/s00216-009-2954-y.

55 U.S. EPA. 2006. Method 8330B (SW-846): Nitroaromatics, Nitramines, and Nitrate Esters by High Performance Liquid Chromatography (HPLC), Revision 2. Washington, DC.

$6{ }^{6} \mathrm{M}$. E. Walsh, Determination of Nitroaromatic, Nitramine, and Nitrate Ester Explosives in Soil by Gas Chromatography and an Electron Capture Detector, Talanta, 2001, 54, 427-438, DOI: 10.1016/S0039-9140(00)00541-5.

77 C. Mullen, A. Irwin, B. V. Pond, D. L. Huestis, M. J. Coggiola and $\mathrm{H}$. Oser, Detection of Explosives and Explosives-Related Compounds by Single Photon Laser Ionization Time-of-Flight Mass Spectrometry, Anal. Chem., 2006, 78, 3807-3814, DOI: 10.1021/ac060190h.

$8{ }^{8}$ A. Bromberg and R. A. Mathies, Homogeneous Immunoassay for Detection of TNT and Its Analogues on a Microfabricated Capillary Electrophoresis Chip, Anal. Chem., 2003, 75, 11881195, DOI: 10.1021/ac020599g.

$9{ }^{9}$ C. K. Chua, M. Pumera and L. Rulíšek, Reduction Pathways of 2,4,6-Trinitrotoluene: An Electrochemical and Theoretical Study, J. Phys. Chem. C, 2012, 116, 4243-4251, DOI: 10.1021/jp209631x.

1010 Y. T. Yew, A. Ambrosi and M. Pumera, Nitroaromatic Explosives Detection Using Electrochemically Exfoliated Graphene, Sci. Rep., 2016, 6, 1-11, DOI: 10.1038/srep33276.

$11{ }^{11}$ H. X. Zhang, A. M. Cao, J. S. Hu, L. J. Wan and S. T. Lee, Electrochemical Sensor for Detecting Ultratrace Nitroaromatic Compounds Using Mesoporous $\mathrm{SiO}_{2}$-Modified
There are no conflicts to declare.

\section{Acknowledgements}

The authors gratefully acknowledge the Klaus Tschira Foundation for financial and administrative support, as well as the state of Baden-Württemberg through bwHPC, the Interdisciplinary Center for Scientific Computing (IWR) of Heidelberg University and the Heidelberg Institute for Theoretical Studies (HITS gGmbH) for computational resources. The authors also thank Dr. Christopher Ehlert and Dr. Michelle Ernst for helpful scientific discussions and methodological assistance, and Dr. John Lindner for proofreading this manuscript.

\section{Notes and references}

¥ The limit of detection (LOD) of a sensor is the minimal concentration of analyte that generates a signal significantly different from the blank; sensitivity of a sensor reflects the change in its response to a small change in stimulus causing the response. ${ }^{41}$

Electrode, Anal. Chem., 2006, 78, 1967-1971, DOI: 10.1021/ac051826s.

1212 M. Pumera, Electrochemistry of Graphene, Graphene Oxide and Other Graphenoids: Review, Electrochem. Commun., 2013, 36, 14-18, DOI: 10.1016/j.elecom.2013.08.028.

$13{ }^{13}$ V. Singh, D. Joung, L. Zhai, S. Das, S. I. Khondaker and S. Seal, Graphene Based Materials: Past, Present and Future, Prog. Mater. Sci., 2011, 56, 1178-1271, DOI: 10.1016/j.pmatsci.2011.03.003.

$14{ }^{14}$ A. Piras, C. Ehlert and G. Gryn'ova, Sensing and Sensitivity: Computational Chemistry of Graphene-Based Sensors, WIREs Comput. Molec. Sci., 2021, Early View, DOI: 10.1002/wcms.1526.

$15{ }^{15}$ C. Anichini, W. Czepa, D. Pakulski, A. Aliprandi, A. Ciesielski and P. Samorì, Chemical Sensing with 2D Materials, Chem. Soc. Rev., 2018, 47, 4860-4908, DOI: 10.1039/c8cs00417j.

$16{ }^{16} \mathrm{G}$. Ersan, O. G. Apul, F. Perreault and T. Karanfil, Adsorption of Organic Contaminants by Graphene Nanosheets: A Review, Water Res., 2017, 126, 385-398, DOI: 10.1016/j.watres.2017.08.010.

1717 T. J. M. Fraga, M. N. Carvalho, M. G. Ghislandi and M. A. da. Motta Sobrinho, Functionalized Graphene-Based Materials as Innovative Adsorbents of Organic Pollutants: A Concise Overview, Braz. J. Chem. Eng., 2019, 36, 1-31, DOI: 10.1590/0104-6632.20190361s20180283.

1818 Y. J. Dappe, M. Andersen, R. Balog, L. Hornekær and X. Bouju, Adsorption and STM Imaging of Polycyclic Aromatic Hydrocarbons on Graphene, Phys. Rev. B, 2015, 91, 45427, DOI: 10.1103/PhysRevB.91.045427.

$19{ }^{19}$ X. Zhou, C. Yuan, D. Qin, Z. Xue, Y. Wang, J. Du, L. Ma, L. Ma and X. Lu, Pd Nanoparticles on Functionalized Graphene for Excellent Detection of Nitro Aromatic Compounds, Electrochim. Acta, 2014, 119, 243-250, DOI: 10.1016/j.electacta.2013.10.197.

$20{ }^{20}$ T. H.; Seah, H. L.; Poh, C. K.; Chua, Z.; Sofer and M. Pumera, Towards Graphane Applications in Security: The Electrochemical Detection of Trinitrotoluene in Seawater on 
Hydrogenated Graphene, Electroanalysis, 2014, 26, 6268, DOI: 10.1002/elan.201300228.

$21{ }^{21}$ B. K. Ong, H. L. Poh, C. K. Chua and M. Pumera, Graphenes Prepared by Hummers, Staudenmaier and Hofmann Methods for Analysis of TNT-Based Nitroaromatic Explosives in Seawater, Electroanalysis, 2012, 24, 2085-2093, DOI: 10.1002/elan.201200474.

2222 R.; Zhang, C.; Zhang, F.; Zheng, X.; Li, C. L. Sun and W. Chen, Nitrogen and Sulfur Co-Doped Graphene Nanoribbons: A Novel Metal-Free Catalyst for High Performance Electrochemical Detection of 2, 4, 6-Trinitrotoluene (TNT), Carbon N. Y., 2018, 126, 328-337, DOI: 10.1016/j.carbon.2017.10.042.

2323 T. W. Chen, J. Y. Xu, Z. H. Sheng, K. Wang, F. B. Wang, T. M. Liang and X. H. Xia, Enhanced Electrocatalytic Activity of Nitrogen-Doped Graphene for the Reduction of Nitro Explosives, Electrochem. Commun., 2012, 16, 30-33, DOI: 10.1016/j.elecom.2011.12.016.

$24{ }^{24}$ S. M. Tan, C. K. Chua and M. Pumera, Graphenes Prepared from Multi-Walled Carbon Nanotubes and Stacked Graphene Nanofibers for Detection of 2,4,6-Trinitrotoluene (TNT) in Seawater, Analyst, 2013, 138, 1700-1704, DOI: 10.1039/c3an00089c.

2525 M. S. Goh and M. Pumera, Graphene-Based Electrochemical Sensor for Detection of 2,4,6- Trinitrotoluene (TNT) in Seawater: The Comparison of Single-, Few-, and Multilayer Graphene Nanoribbons and Graphite Microparticles, Anal. Bioanal. Chem., 2011, 399, 127-131, DOI: 10.1007/s00216010-4338-8.

2626 J. Park, Y. Kim, S. Y. Park, S. J. Sung, H. W. Jang and C. R. Park, Band Gap Engineering of Graphene Oxide for Ultrasensitive $\mathrm{NO}_{2}$ Gas Sensing, Carbon, 2020, 159, 175-184, DOI: 10.1016/j.carbon.2019.11.063.

2727 S. J. Rodríguez, L. Makinistian and E. A. Albanesi, Graphene for Amino Acid Biosensing: Theoretical Study of the Electronic Transport, Appl. Surf. Sci., 2017, 419, 540-545, DOI: 10.1016/j.apsusc.2017.05.031.

$28{ }^{28}$ S. J. Rodríguez and E. A. Albanesi, Electronic Transport in a Graphene Single Layer: Application in Amino Acid Sensing, Phys. Chem. Chem. Phys., 2019, 21, 597-606, DOI: 10.1039/C8CP05093G.

2929 X. Chen and B. Chen, Macroscopic and Spectroscopic Investigations of the Adsorption of Nitroaromatic Compounds on Graphene Oxide, Reduced Graphene Oxide, and Graphene Nanosheets, Environ. Sci. Technol., 2015, 49, 6181-6189, DOI: 10.1021/es5054946.

$30{ }^{30}$ S. Angizi, M. Khalaj, S. A. A. Alem, A. Pakdel, M. Willander, A. Hatamie and A. Simchi, Review-Towards the TwoDimensional Hexagonal Boron Nitride (2D h-BN) Electrochemical Sensing Platforms, J. Electrochem. Soc., 2020, 167, 126513, DOI: 10.1149/1945-7111/abaf29.

3131 F. Neese, Software Update: The ORCA Program System, Version 4.0, WIREs Comput. Mol. Sci., 2018, 8, e1327, DOI: $10.1002 /$ wcms.1327.
$32{ }^{32} \mathrm{~S}$. Grimme, Exploration of Chemical Compound, Conformer, and Reaction Space with Meta-Dynamics Simulations Based on Tight-Binding Quantum Chemical Calculations, J. Chem. Theory Comput., 2019, 15, 2847-2862, DOI: 10.1021/acs.jctc.9b00143.

$33{ }^{33}$ B. Jeziorski, R. Moszynski and K. Szalewicz, Perturbation Theory Approach to Intermolecular Potential Energy Surfaces of van Der Waals Complexes, Chem. Rev., 1994, 94, 18871930, DOI: 10.1021/cr00031a008.

$34{ }^{34}$ E. G. Hohenstein, R. M. Parrish, C. D. Sherrill, J. M. Turney and H. F. Schaefer, Large-Scale Symmetry-Adapted Perturbation Theory Computations via Density Fitting and Laplace Transformation Techniques: Investigating the Fundamental Forces of DNA-Intercalator Interactions, J. Chem. Phys., 2011, 135, 174107, DOI: 10.1063/1.3656681.

3535 R. M. Parrish, L. A. Burns, D. G. A. Smith, A. C. Simmonett, A. E. DePrince, E. G. Hohenstein, U. Bozkaya, A. Yu. Sokolov, R. Di Remigio, R. M. Richard, J. F. Gonthier, A. M. James, H. R. McAlexander, A. Kumar, M. Saitow, X. Wang, B. P. Pritchard, P. Verma, H. F. Schaefer, K. Patkowski, R. A. King, E. F. Valeev, F. A. Evangelista, J. M. Turney, T. D. Crawford and C. D. Sherrill, PsI4 1.1: An Open-Source Electronic Structure Program Emphasizing Automation, Advanced Libraries, and Interoperability, J. Chem. Theory Comput., 2017, 13, 31853197, DOI: 10.1021/acs.jctc.7b00174.

$36{ }^{36}$ E. Pastorczak and C. Corminboeuf, Perspective: Found in Translation: Quantum Chemical Tools for Grasping NonCovalent Interactions, J. Chem. Phys., 2017, 146, 120901, DOI: 10.1063/1.4978951.

3737 P. de Silva and C. Corminboeuf, Simultaneous Visualization of Covalent and Noncovalent Interactions Using Regions of Density Overlap, J. Chem. Theory Comput., 2014, 10, 37453756, DOI: 10.1021/ct500490b.

3838 G. te Velde, F. M. Bickelhaupt, E. J. Baerends, C. Fonseca Guerra, S. J. A. van Gisbergen, J. G. Snijders and T. Ziegler, Chemistry with ADF, J. Comput. Chem., 2001, 22, 931-967, DOI: 1 0.1002/jcc.1056; ADF 2019.304, SCM, Theoretical Chemistry, Vrije Universiteit, Amsterdam, The Netherlands, http://www.scm.com.

3939 H. L. Poh, F. Šaněk, A. Ambrosi, G. Zhao, Z. Sofer and M. Pumera, Graphenes Prepared by Staudenmaier, Hofmann and Hummers Methods with Consequent Thermal Exfoliation Exhibit Very Different Electrochemical Properties, Nanoscale, 2012, 4, 3515-3522, DOI: 10.1039/c2nr30490b.

$40{ }^{40}$ B. Trzaskowski, L. Adamowicz, W. Beck, K. Muralidharan and P. A. Deymier, Impact of Local Curvature and Structural Defects on Graphene-C60 Fullerene Fusion Reaction Barriers, J. Phys. Chem. C, 2013, 117, 19664-19671, DOI: 10.1021/jp405301e.

4141 IUPAC Compendium of Chemical Terminology: Gold Book, 2.1.0.; M. Nič, J. Jirát, B. Košata, A. Jenkins, A. McNaught, Eds.; IUPAC: Research Triagle Park, NC, 2009. https://doi.org/10.1351/goldbook. 